\title{
Contribuições do instrumento “Global Appraisal of Individual Needs” para a assistência e pesquisa: revisão da literatura*
}

\section{Contributions of the instrument "Global Appraisal of Individual Needs” to treatment and research: literature rewiew}

\author{
Heloísa Barboza Paglione ${ }^{1}$, Heloísa Garcia Claro², Márcia \\ Aparecida Ferreira de Oliveira ${ }^{3}$, Janet Titus ${ }^{4}$, Mayara Lima ${ }^{5}$, Thais \\ Correia Pereira ${ }^{5}$
}

\begin{abstract}
PAGLIONE, H. B.; CLARO, H. G.; OLIVEIRA, M. A. F.; TITUS, J.; LIMA, M.; PEREIRA, T. C. Contribuições do instrumento "Global Appraisal of Individual Needs" para a assistência e pesquisa: revisão da literatura. Rev. Ter. Ocup. Univ. São Paulo, v. 23, n. 1, p. 81-88, jan./abr. 2012.

RESUMO: Objetivo: Analisar as contribuições do instrumento "Global Appraisal of Individual Needs" (GAIN), para tratamento e pesquisas sobre a temática do uso de álcool e outras drogas. Métodos: revisão integrativa na base de dados PUBMED. Resultados: O instrumento possui bons índices de validade e confiabilidade, mostrando-se baseado em evidências, e contribui para a assistência e pesquisas relacionadas ao uso prejudicial de álcool e outras drogas, uma vez que é capaz de identificar diversos problemas psicossociais principalmente entre adolescentes e adultos. O GAIN consiste em uma série de mensurações, feitas para apoiar de forma progressiva práticas terapêuticas, incluindo análise inicial, intervenções breves, auxílio a diagnósticos, entre outros que possibilitarão um tratamento focalizado nas necessidades do indivíduo.
\end{abstract}

DESCRITORES: Álcoolismo; Drogas ilícitas; Prática clínica baseada em evidências; Literatura de revisão como assunto; Avaliação de resultado de intervenções terapêuticas/métodos.

\footnotetext{
*Apoio financeiro do Conselho Nacional de Desenvolvimento Científico e Tecnológico (CNPq).

1. Estudante do curso de Licenciatura em Enfermagem da Escola de Enfermagem da USP.

2. Doutoranda pelo Programa de Pós Graduação em Enfermagem da Escola de Enfermagem da USP

3. Docente do Departamento de Enfermagem Materno Infantil e Psiquiátrica da Escola de Enfermagem da USP.

4. Pesquisadora no Centro de Coordenação do GAIN, Chestnut Health Systems.

5. Enfermeira no Serviço de Atenção Integral ao Dependente - SAID.

Endereço para correspondência: Av. Dr. Enéas de Carvalho Aguiar, 419. CEP: 05403-000 - São Paulo, SP. e-mail: heloisa.paglione@ usp.br
} 


\section{INTRODUÇÃO}

$\mathrm{N}$

o Brasil, aproximadamente $12,3 \%$ da população pode ser considerada dependente de álcool, com prevalência de $17,1 \%$ entre a população masculina e 5,7\% na feminina. A dependência alcoólica apresenta alta prevalência quando comparada com outras doenças e representa um dos maiores problemas de saúde pública no Brasil (CARLINI et al., 2005; GALLASSI et al., 2008).

Já em relação às drogas ilegais, uma em cada cinco pessoas que faz uso desse tipo de substância pode ser diagnosticada como dependente. As funções ligadas a percepção, humor e consciência são afetados pelo uso de substâncias psicoativas, e isso pode influenciar a capacidade em exercer controle sobre seu uso de drogas e resultar em dependência física e psicológica, que constrange o indivíduo a continuar o uso apesar das conseqüências adversas. Além das incapacidades e perda da saúde física, as pessoas com transtornos causados pelo uso de álcool e outras drogas podem sofrer de problemas psicológicos, psicossociais, problemas interpessoais, perda de emprego, dificuldades de aprendizado, além de problemas legais, dentre eles atividades ilícitas para aquisição da substância de consumo, participação no tráfico, entre outros (GALLASSI et al., 2008).

A Organização Mundial da Saúde (OMS) estima que, no mundo, 151 milhões de pessoas sofram de depressão, 26 milhões de esquizofrenia, 125 milhões são afetadas por uso de álcool e outras drogas, 40 milhões sofrem de epilepsia e 24 milhões de Alzheimer e outras demências. Cerca de 844 mil pessoas morrem por suicídio a cada ano (FUNK et al., 2010).

Apesar do vasto conhecimento sobre os impactos, mentais, psicológicos e sociais do uso de álcool e outras drogas na vida dos indivíduos, existem poucos instrumentos capazes de levantar os problemas dos indivíduos em todas as áreas atingidas. De modo que se recorre a diversas e distintas avaliações: avaliação do estado mental, classificação do uso de drogas, avaliação sobre envolvimento em problemas judiciais e criminais, e assim sucessivamente. Este processo dispende tempo, dinheiro e outros recursos públicos e privados, podendo tornar a coleta de informações acerca das necessidades do indivíduo um processo assistemático e não eficiente.

A avaliação pode fornecer informações cruciais a respeito dos pacientes, seus problemas, histórias de vida, e potencialidades. Uma avaliação pode identificar se o indi- víduo apresenta problemas com uso de substâncias e sobre a severidade destes problemas. Instrumentos são úteis na prática dos serviços de saúde, embora, na maior parte dos cenários de cuidado à saúde, não estão disponíveis instrumentos abrangentes, capazes de produzir uma avaliação multissistêmica. As avaliações únicas são consideradas difíceis para a rotina dos serviços de saúde. Considera-se também que para a coleta de todas as informações são necessárias inúmeras fontes de informação e a opinião de diversos profissionais (JOHNSON, 2004).

A realização de diversas avaliações, uma para cada aspecto do paciente, feitas por profissionais diferentes, pode resultar em informações desencontradas, repetidas, e não assegura a possível omissão de informações relevantes. A organização dos dados e informação sobre o pacientes pode ser de extrema importância para a qualidade do cuidado. Problemas na utilização das avaliações dificultam o cuidado ao pacientes, bem como as investigações para o avanço das práticas de saúde (JOHNSON, 2004; DELVAN et al., 2010).

Frente à problemática apresentada acima, pesquisadores desenvolveram o Global Appraisal of Individual Needs - Initial - GAIN-I. Este instrumento possui 103 escalas e índices que avaliam os problemas, mensuram mudanças e documentam o uso de serviços e se mostrou válido para adolescentes e adultos.

O GAIN-I articula aspectos e sintomas relacionados ao uso de substâncias que podem ser encontrados no "Manual Diagnóstico e Estatístico de Distúrbios Mentais" (DSM IV). (JOHNSON, 2004; DENNIS et al., 2006; CLARO; OLIVEIRA, 2010).

Portanto, frente à demanda mundial de instrumentos e práticas facilitadoras do cuidado em álcool e drogas, este estudo objetiva analisar as contribuições, presentes na literatura internacional, do instrumento Global Appraisal of Individual Needs para a assistência e estimular investigações que objetivem a adaptação e validação desta ferramenta para a realidade Brasileira.

\section{OBJETIVOS}

Analisar os estudos publicados na base de dados PubMed, que tomaram como objeto o uso, aplicações e aspectos relacionados aos instrumentos da família $\operatorname{GAIN}^{(1)}$, entre os anos de 2000 a 2010. Descrever as possíveis contribuições dos instrumentos GAIN para a assistência ao indivíduo que faz uso prejudicial de álcool e outras drogas

(1) A Família de instrumentos GAIN se refere às diversas versões do instrumento, idealizadas para fases distintas do tratamento. Estas versões estão expostas na Figura 1 deste artigo. 


\section{MÉTODOS}

A revisão bibliográfica é um tipo de estudo que permite que o conhecido sobre um determinado assunto seja identificado, analisado e apresentado de forma sucinta ressaltando sua relevância (DELVAN et al., 2010). A revisão integrativa permite que a literatura seja analisada de forma ampla, englobando estudos experimentais e não experimentais, tendo como finalidade reunir e sintetizar o conhecimento já produzido sobre o tema investigado, ou seja, permite buscar, avaliar e sintetizar as evidências disponíveis para a sua incorporação na prática. $\mathrm{O}$ conhecimento gerado por meio da revisão integrativa pode ser utilizado no campo das políticas de saúde, embasando a prática baseada em evidências (SILVEIRA, 2005).

Esta revisão integrativa foi realizada em seis etapas, que serão descritas a seguir.

\section{Primeira etapa: definição do tema e da questão norteadora}

Por meio de uma análise preliminar da literatura percebeu-se a importância de formular intervenções baseadas em evidências, para realizar diagnóstico e planejamento do tratamento de indivíduos em situação de risco, que fazem uso abusivo de álcool e outras drogas, ou são dependentes dessas substâncias. Para isto, é necessário levantar informações a respeito das necessidades desta população. Os instrumentos da família GAIN são identificados na literatura como instrumentos que possibilitam estes processos, porém, percebeu-se a necessidade de realizar um levantamento de dados sistemático acerca das contribuições e aplicabilidade do instrumento GAIN, Partiu-se do seguinte questionamento: quais são as informações disponíveis na literatura acerca das características principais dos instrumentos da família GAIN, suas propriedades psicométricas, sua aplicabilidade prática e contribuição para pesquisas científicas?

\section{Segunda etapa: busca bibliográfica}

A revisão bibliográfica foi realizada na base de dados Pubmed. Definiu-se os anos 2000 e 2010, porém só foram encontrados artigos publicados nos período de 2002 a 2010. Foi utilizado para a busca o descritor: Global Appraisal of Indivual Needs e obtidos 38 artigos. Desse total, foram excluídos 26 artigos por não se tratar de estudos baseados no instrumento, o que resultou em uma amostra de 12 artigos. Os artigos que não possuíam versão completa on-line foram obtidos por meio de contato com os autores e periódicos via $e$-mail e correio.

Face à pouca disponibilidade de materiais na base de dados consultada selecionou-se também outros materiais bibliográficos: os manuais dos instrumentos e outros documentos publicados pelos desenvolvedores do instrumento, identificados nas referências dos artigos encontrados, obtendo-se mais 7 materiais. A amostra de final foi composta por 19 itens.

\section{Terceira etapa: categorização}

Para facilitar a análise dos dados, as informações consideradas relevantes aos objetivos da pesquisa foram reunidas em fichas. Cada ficha continha campos para preenchimento de informações acerca de características gerais do instrumento, suas propriedades psicométricas, qualidade dos dados colhidos, informações a respeito de estudos realizados utilizando-se do instrumento GAIN como meio para coleta de dados, informações a respeito de dificuldades e limites do instrumento quando adaptado para uso em outras culturas, entre outras consideradas importantes para se atingir o objetivo deste estudo.

\section{Quarta etapa: avaliação dos estudos}

As publicações selecionadas e catalogadas foram lidas na íntegra, e suas respectivas fichas foram submetidas a releitura para garantir a presença de informações relevantes.

\section{Quinta e sexta etapas: síntese do conhecimento e interpretação e discussão \\ Nestas etapas os dados foram submetidos a análise comparativa, tendo sido organizados quadros para facilitar a avaliação dos resultados e sintetizar o conhecimento adquirido, tornando-o acessível. Por meio da análise dos quadros, foi possível levantar informações relevantes acerca das contribuições dos instrumentos da família GAIN, para a assistência e pesquisa, apresentadas na seção a seguir.}

\section{RESULTADOS E DISCUSSÃO}

Os 19 artigos inicialmente encontrados na busca bibliográfica têm como autores pesquisadores dos EUA. Este resultado indica que ainda não foram realizados muitos estudos nas regiões para as quais o instrumento foi adaptado e validado. Quanto ao ano de publicação todos os artigos foram produzidos a partir do ano de 2002.

As informações obtidas foram divididas nas seguintes categorias: características do instrumento - $\mathrm{O}$ GAIN; populações com as quais o GAIN pode ser utilizado; propriedades Psicométricas.

\section{Características do Instrumento - O GAIN}

Os instrumentos GAIN são instrumentos de 
mensuração psicossocial que buscam integrar estado clínico e evidências da pesquisa científica, em uma entrevista estruturada em oito seções principais (antecedentes, uso de substâncias, saúde física, comportamentos de risco, saúde mental, ambiente, aspectos legais e potencialidades vocacionais) (DENNIS et al., 2006).

O GAIN-I é descrito como um instrumento para uso em pesquisas sobre uso de álcool e drogas, bem como para realização de diagnóstico, planejamento do tratamento, avaliação da evolução do paciente, entre outros aspectos relacionados ao cuidado de adolescentes usuários de álcool e outras drogas(Dennis et al., 2004, 2006).

$\mathrm{O}$ instrumento possui 103 escalas e índices que avaliam os problemas, mensuram mudanças e documentam o uso de serviços. O GAIN-I articula aspectos e sintomas relacionados ao uso de substâncias que podem ser encontrados no "Manual Diagnóstico e Estatístico de Distúrbios Mentais" (DSM IV)(DENNIS et al., 2006).

$\mathrm{Na}$ administração do instrumento, o entrevistador e o paciente trabalham juntos: o entrevistador solicita por diversas vezes que o paciente estime dados como o número de dias/horas que passou utilizando drogas nos últimos meses, a quantidade de droga utilizada, bem como outros dados relacionados ao consumo. As questões feitas para o paciente são simples, porém, em caso de dúvidas, o entrevistador pode fornecer explicações, com a condição de que não sejam feitas mudanças ou prejuízos no significado do item. O paciente pode também recusar-se a responder quaisquer perguntas com as quais não se sinta a vontade ou para as quais não saiba a resposta (DENNIS et al., 2004).

Durante a entrevista, são oferecidos intervalos ao paciente. Esses intervalos são importantes uma vez que a entrevista pode ser longa (90 a 120 minutos) e o indivíduo pode não responder de forma apropriada. Alguns estudos apontam que quando os intervalos são oferecidos, o tempo total de aplicação é diminuído, uma vez que os pacientes são capazes de compreender melhor as questões, fornecendo melhores respostas (GOTHAM et al., 2008).

Os aspectos relativos ao paciente, levantados pelo instrumento, geram uma dimensão dos sintomas que conta com mensurações e impressões de diagnósticos em quatro principais tópicos de interesse: a) efeitos internos; aspectos relacionados à depressão, ansiedade, estresse traumático, suicídio. b) efeitos externos: déficit de atenção, distúrbio de hiperatividade, desvio de conduta. c) problemas relacionados a crimes ou outras complicações legais com as quais o paciente possa estar envolvido, e situações de violência. d) severidade do uso de substâncias (DENNIS et al., 2003, 2006).

Desta forma, os problemas sentidos e relatados pelo paciente, em cada um destes tópicos de interesse, são contabilizados por meio do instrumento e são gerados diagnósticos baseados no DSM-IV e no CID-10 - Classificação Internacional de doenças.

As informações levantadas permitem traçar um perfil amplamente estruturado, o que pode subsidiar intervenções individualizadas, eficientes e que são mais adequadas ao perfil psicossocial em questão (DENNIS et al., 2006).

Para sua aplicação o GAIN-I necessita de alguns cuidados específicos, como o treinamento profissional e um período relativamente longo junto ao paciente. $\mathrm{O}$ treinamento e avaliação duram cerca de 2 a 3 meses e a aplicação do instrumento leva em torno de 90 a 120 minutos.

Apesar de o instrumento ser eficiente na coleta de informações, são necessárias de 2 a 4 horas para completar todo o processo de aplicação, incluindo exames laboratoriais relacionados, avaliações de saúde mental e preenchimento de outros documentos. Assim, surgiu a necessidade de novas estratégias para a coleta de dados, que tornassem o processo mais rápido e eficiente (RILEY et al., 2007).

Há um grande número de usuários de álcool e outras drogas que apresentam outros transtornos mentais. Indivíduos com múltiplos diagnósticos estão mais propensos a experimentar problemas com o tratamento, não aderência à medicação, maior período de permanência no serviço, menor qualidade de vida e piores resultados no tratamento pelo uso de substancias. O GAIN Short Screener (GAIN-SS) é um instrumento que leva de 3 a 5 minutos para identificar pessoas que possuam comorbidades o que propicia o encaminhamento do indivíduo para serviços e tratamento adequados ao seu perfil. O GAIN-SS é uma avaliação inicial para triagem e não substitui a avaliação mais detalhada. Por sua eficiência e fácil implementação, o GSS pode ser utilizado na escola, no local de trabalho ou no sistema de justiça interessado na detecção precoce e implantação de intervenção, bem como em serviços de atenção básica, pronto-socorros, entre outros (DENNIS et al., 2006).

Foram realizadas análises psicométricas provando que as versões reduzidas do instrumento (GAIN-SS e GAIN-Q - GAIN R - GAIN Rápido) são confiáveis, mostrando correspondência próxima com as versões completas (GAIN-I e GAIN-M90 Monitoração após 90 dias de tratamento). A versão completa e a versão reduzida mostram uma convergência similar em termos de índices de validade e confiabilidade (TITUS et al., 2008).

Os artigos colocam como importante limitação dos instrumentos GAIN o auto-relato dos participantes, sendo necessário o uso de exames complementares e outras coletas de dados para validar as informações. Verifica-se que o GAIN-I pode facilitar a detecção de comportamentos de 
risco, uso abusivo, dentre outros aspectos relacionados ao uso de álcool e drogas fornecer informações para estratégias de prevenção primária e secundária nos serviços de saúde e auxiliar no planejamento do tratamento e intervenções individualizadas.

O GAIN é descrito como instrumento capaz de produzir o diagnóstico minucioso acerca do uso de drogas e aspectos relacionados ao uso. Os benefícios deste diagnóstico vão desde um melhor planejamento do tratamento, baseado nas necessidades individuais de cada paciente, à prevenção de agravos psicossociais decorrentes do uso de álcool e outras drogas. Existem diversas versões do GAIN, conforme exposto na Figura 1, abaixo, cada uma projetada para fornecer uma abordagem progressiva para a avaliação, em diferentes momentos, em distintos contextos e para diferentes populações. Foi possível fazer uma breve descrição de cada um destes instrumentos, conforme é apresentada nos dados da Figura 1.

Figura 1. Versões do GAIN e tempo necessário para administração

\begin{tabular}{llc}
\hline Nome/ Tradução/ Sigla & Características & $\begin{array}{c}\text { Tempo de Administração } \\
\text { (em minutos) }\end{array}$ \\
\hline $\begin{array}{l}\text { GAIN Initial/ GAIN inicial/ } \\
\text { GAIN-I }\end{array}$ & Avaliação biopsicossocial completa & 120 \\
$\begin{array}{l}\text { GAIN monitoring 90 days/GAIN mo- } \\
\text { nitoração de } 90 \text { dias./ GAIN M90. }\end{array}$ & Instrumento de acompanhamento trimestral & 45 - 60 \\
$\begin{array}{l}\text { GAIN Quick/ GAIN Rápido/ GAIN } \\
\text { - Q3. }\end{array}$ & $\begin{array}{l}\text { Subconjunto de itens do GAIN-I. } \\
\text { Pode ser utilizado para subsidiar intervenções breves. }\end{array}$ & 20 - 30 \\
$\begin{array}{l}\text { GAIN-Q-Monitoring 90 Days. GAIN } \\
\text { acompanhamento de } 90 \text { dias/ GAIN }\end{array}$ & $\begin{array}{l}\text { Monitora resultados trimestrais. Aplicação mais rápida } \\
\text { que o GAIN M90. }\end{array}$ & 15 - 20 \\
$\begin{array}{l}\text { GAIN Short Screener/GAINAvaliação } \\
\text { breve/ GAIN-SS. }\end{array}$ & Instrumento de rastreamento & 5 \\
$\begin{array}{l}\text { Treatment Satisfaction Index. Índice de } \\
\text { satisfação com o tratamento. TxSI. }\end{array}$ & Mede a relação de trabalho entre a equipe de atendimento & 5 \\
\hline
\end{tabular}

\section{Características da população com a qual o GAIN pode ser utilizado}

Os artigos selecionados demonstraram o uso satisfatório dos instrumentos GAIN em adolescentes usuários e dependentes de álcool e outras drogas, mas também em adultos jovens e adultos (PERRY et al., 2004; DENNIS et al., 2006).

O instrumento GAIN também foi utilizado com pacientes que apresentam comorbidades de outros transtornos mentais, como em casos de depressão associada, ideação suicida, entre outros, assim como com adolescentes em conflito com a lei, tanto em serviços de atendimento ambulatorial como em serviços de internação (SUBRAMANIAM et al., 2007; CHAN et al., 2008; RAMCHAND et al., 2009; CONRAD et al., 2010).

Alguns dos estudos selecionados descrevem a aplicação do instrumento em grupos, sendo a maior parte dos indivíduos do sexo masculino, adolescentes, que são usuários prejudiciais ou dependentes de álcool e outras drogas e que, em média, iniciaram o uso de substancias com 12 anos de idade (DENNIS et al., 2004).

Os adultos, usuários de álcool e outras drogas, descritos pelos estudos nos quais os instrumentos GAIN foram utilizados apresentaram maior propensão a comorbidades tais como tendência a desordens como depressão, ansiedade, entre outros e diagnóstico de dependência de substâncias. Já os adolescentes apresentaram maior tendência a abuso (e não dependência) de substancias, desordens como déficit de atenção, distúrbio de hiperatividade e desvio de conduta e questões relacionadas a crime e violência. Os indivíduos que foram submetidos a entrevistas com o uso dos instrumentos GAIN apresentados nos estudos pertencem a grupos heterogêneos, e, como observado na próxima seção deste artigo, possuíram boas propriedades psicométricas, sugerindo que o instrumento pode ser amplamente utilizado 
(DENNIS et al., 2006).

\section{Pontuação e propriedades psicométricas do GAIN}

Para descrição da pontuação e propriedades psicométricas do GAIN, nos basearemos no instrumento completo, o GAIN-I, uma vez que as outras versões são derivadas deste instrumento. Todos os artigos encontrados fazem menção aos scores do GAIN.

\section{Pontuação}

São utilizados métodos diferentes de pontuação das respostas do indivíduo. Algumas escalas possuem opções de resposta de Sim (1) ou Não (0), porém, também são utilizadas escalas somatórias, que atribuem números conforme as opções escolhidas (DENNIS et al., 2002, 2003).

\section{Propriedades Psicométricas do GAIN-I}

O GAIN-I possui 103 escalas e as principais possuem coeficiente alfa $(\alpha)$ superior a 0.9 e as subscalas normalmente possuem $\alpha$ superiores a 0.7 . A consistência interna dessas escalas clássicas foram consideradas de bom a ótimo em estudos com populações que variaram por gênero, raça, idade e local de origem, assim como por níveis de cuidado e inúmeros subgrupos clínicos como mulheres grávidas, usuários de drogas injetáveis, moradores de rua ou pessoas com comorbidades de distúrbios mentais (DENNIS et al., 2003).

Usando dados de 1028 adolescentes de 14 ambulatórios e programas residenciais, foi apurado que as escalas do GAIN-I replicam os resultados com alta consistência interna nas summary dimension escales (escalas resumo das dimensões) e nas subscalas especificas: a) Escala de Problemas com Substâncias (16 itens $/ \alpha=0.9$ ) e suas subscalas: Índice de Questões decorrentes de Substâncias $(5 / 0,67)$, Índice de Abuso de Substância $(4 / 0,70)$, Escala de Dependência de Substância (7/ 0,83), e Escala de Distúrbio por Uso de Substância (11/0,87); b) Escala de Sofrimento Mental Interno (39 itens, $\alpha=0,94$ ) e suas subscalas: Índice de Sintomas Somáticos $(4 / 0,69)$, Escala de Sintomas Depressivos $(6 / 0,77)$, Escala de Pensamentos Homicidas e Suicidas $(4 / 0,83)$, Escala de Sintomas de Medo e Ansiedade (10/0,77), Escala de Estresse Traumático (13/0,92) e Escala de Sofrimento Mental Geral $(21 / 0,88)$; c) Escala de Comportamento Complexo (33 itens $/ \alpha=0,91$ ) e suas subscalas: Escala de desordem e desatenção $(9 / 0,88)$, Escala de Hiperatividade e Impulsividade $(9 / 0,81)$, Escala de Desordem de Conduta $(15 / 0,82)$, Escala Transtorno de Déficit de Atenção (18/0,90); d) Escala de Crime e Violência (22 itens/ $\alpha=0,9)$ e suas subscalas: Escala Tática de Conflito Geral
$(12 / 0,89)$, Escala de Crime Contra Propriedade $(6 / 0,75)$, Escala Interpessoal de Crime (7/0,67), Escala de Crimes por Drogas $(4 / 0,53)$ e Escala Geral de Crime $(17 / 0,84)$ (Dennis, White et al., 2003).

Visando analisar a validade da mensuração proposta pelo GAIN-I, diferentes métodos que reportam o uso de substância foram comparados (auto relato, urina e saliva) para determinar a validade dos itens de auto-resposta e cada método foi consistente com a estimativa combinada de qualquer uso. A estatística kappa foi de 0,59 para autorelato, 0,69 para urina, 0,56 para saliva. Em relação à confiabilidade da medida, o GAIN-I demonstrou-se confiável num estudo de teste-reteste no qual obteve um coeficiente de correlação de Spearman (rho) de 0,94. Ademais, diagnósticos baseados no GAIN tiveram confiabilidade consistente em estudo de teste-reteste para distúrbios decorrentes do uso de substâncias ao possuírem kappa 0,60 (LENNOX et al., 2006).

Além disso, usando análise discriminante, as escalas do GAIN-I foram capazes de estabelecer com precisão diagnósticos psiquiátricos independentes por uma equipe em estudo cego de comorbidades psiquiátricas, dentre as quais Transtorno de Déficit de Atenção (kappa $=1.00$ ), Transtornos de Humor (kappa = .85), Desvios de Conduta/ Transtorno Desafiador de Oposição (kappa = .82), Transtornos de Ajustamento (kappa = .69), ou a falta de diagnóstico de não uso de substância (kappa $=.91)$ e foram capazes de discriminar as outras desordens primárias transversalmente a estas condições (kappa =.65). Estudos feitos com adultos e adolescentes encontraram boa confiabilidade em estudos teste-reteste em termos da freqüência do uso ( $\mathrm{r}=$ 0,7 a 0,8 ) assim como na necessidade de tratamento (kappa $=0,78)$. Auto-relatos foram consistentes com os relatos dos pais, testes com urinas e salivas (kappa de 0,5 a 0,8). Ademais, auto-relatos foram consistentes com estimativas multi-métodos baseados em qualquer auto-relato ou testes positivos para urina e saliva para qualquer droga (kappa $=$ $0,56)$, cocaína $($ kappa $=0,52)$, opiáceos $($ kappa $=0,55)$ e maconha $(\mathrm{kappa}=0,75)$. Nenhum método foi superior em todas as drogas (DENNIS et al., 2003).

Outro estudo encontrado avaliou a confiabilidade e a validade do GAIN comparativamente com o TimeLine FollowBack (TLFB). Ambos instrumentos podem ser utilizados para levantar dados a respeito do número de dias e quantidades de uso de álcool e cannabis Sativa L. Na análise realizada entre os dois instrumentos, foi observado que eles têm validade similar nos termos de relacionamento com os sintomas de abuso ou dependência (DENNIS et al., 2004).

Considerando as propriedades psicométricas descri- 
tas o instrumento GAIN-I possui bons índices de validade e confiabilidade, tanto para uso com adultos como para uso com adolescentes. Instrumentos como este são necessários para o cuidado e prevenção ao uso de álcool e outras drogas, principalmente em se tratando de populações de difícil manejo como adolescentes, uma vez que possuem características como desmotivação, mal-estar e até mesmo irritação que podem dificultar o cuidado.

\section{CONCLUSÕES}

Verifica-se que os instrumentos GAIN podem contribuir para assistência a indivíduos usuários de álcool e outras drogas. Estas contribuições são, principalmente, no sentido de facilitar a detecção de comportamentos de risco, uso abusivo, dentre outros aspectos relacionados ao consumo de álcool e drogas. Fornece informações para estratégias de prevenção primária e secundária, nos serviços de saúde, e auxiliar no planejamento do tratamento e de intervenções individualizadas. Conclui-se que o instrumento pode beneficiar diversas populações uma vez que os estudos apontam bons coeficientes de validade e confiabilidade para adolescentes e adultos.

Os dados encontrados mostram que os instrumentos GAIN são um meio de avaliação baseado em evidências e, considerando-se que o campo de tratamento para indivíduos que fazem uso abusivo de álcool e outras drogas demanda práticas baseadas em evidências (PBE), este pode trazer contribuições também para outros países e culturas. Entretanto, é clara a necessidade de estudos das regiões onde o instrumento é utilizado atualmente, dado que os estudos são oriundos apenas dos EUA.

PAGLIONE, H. B.; CLARO, H. G.; OLIVEIRA, M. A. F.; TITUS, J.; LIMA, M.; PEREIRA, T. C. Contributions of the instrument "Global Appraisal of Individual Needs" to treatment and research: literature rewiew. Rev. Ter. Ocup. Univ. São Paulo, v. 23, n. 1, p. 81-88, jan./abr. 2012.

\begin{abstract}
S: Objective: To analyze the literature searching for contributions of the instrument "Global Appraisal of Individual Needs" - GAIN for treatment and research on the topic of alcohol and other drugs. Methods: integrative review on the PUBMED database. Results: the instrument has good levels of validity and reliability, being based on evidence, and contributes to the care and research related to the harmful use of alcohol and other drugs because it is able to identify several psychosocial problems especially among adolescents and adults. The GAIN is a series of measurements made in support of a progressive therapy practices, including initial assessment, brief interventions, to aid diagnosis, and other factors that will enable a practice focused on the needs of individual.
\end{abstract}

KEYWORDS: Alcoholism; Street drugs; Evidence-based practice; Review literature as topic; Evaluation of results of therapeutics interventions/methods.

\title{
REFERÊNCIAS
}

CARLINI, E.; GALDURÓZ, J.; NOTO, A.; CARLINI, C.; OLIVEIRA, L.; NAPPO, A. II levantamento domiciliar sobre o uso de drogas psicotrópicas no Brasil: envolvendo as 108 maiores cidades do país. In: CENTRO BRASILEIRO DE INFORMAÇÃO SOBRE DROGAS - CEBRID. Drogas. Brasília. DF, 2005.

CHAN, Y.; DENNIS, M.; FUNK, R. Prevalence and comorbidity of major internalizing and externalizing problems among adolescents and adults presenting to substance abuse treatment. J. Subst. Abuse Treat., 34, 14-24. 2008

CLARO, H. G.; OLIVEIRA, M. A. F. D. Adaptação cultural do instrumento "Global Appraisal of Individual Needs" para uso com adolescentes no Brasil. [Projeto de Mestrado] - Escola de Enfermagem da Universidade de São Paulo, São Paulo, 2010.

CONRAD, K.; BEZRUCZKO, N.; CHAN, Y.; RILEY, B.; DIAMOND, G.; DENNIS, M. Screening for atypical suicide risk with person fit statistics among people presenting to alcohol and other drug treatment. Drug Alcohol Depend., v. 106, p. 92-100, 2010 .

DELVAN, J. D. S.; PORTES, J. R. M.; CUNHA, M. P.; MENEZES, M.; LEGAL, E. J. Children who use mental health services: characterization of the population in a city in southern Brazil. Rev. Bras. Crescimento Desenvolv. Hum., v. 20, p. 228, 2010. 
DENNIS, M.; CHAN, Y.; FUNK, R. Development and validation of the GAIN Short Screener (GSS) for internalizing, externalizing and substance use disorders and crime/violence problems among adolescents and adults. Am. J. Addict., v. 15, Suppl. 1, p. 80-91, 2006.

DENNIS, M.; DAWUD-NOURSI, S.; MUCK, R.; MCDERMEIT, M. The need for developing and evaluating adolescent treatment models. Adolescent substance abuse treatment in the United States: Exemplary Models from a National Evaluation Study. Binghamton, NY: Haworth Press, 2002. p. 3-34.

DENNIS, M.; WHITE, M.; TITUS, J.; UNSICKER, J. Global Appraisal of Individual Needs: Administration Guide for the GAIN and related Measures. In: Bloomington, 2003.

DENNIS, M.; FUNK, R.; GODLEY, S.; GODLEY, M.; WALDRON, H. Cross-validation of the alcohol and cannabis use measures in the Global Appraisal of Individual Needs (GAIN) and Timeline Followback (TLFB; Form 90) among adolescents in substance abuse treatment. Addiction, v. 99, Suppl. 2, p. 120128,2004

FUNK, M.; DREW, N.; FREEMAN, M.; FAYDI, E.; World Health Organization. Mental health and development: targeting people with mental health conditions as a vulnerable group. Geneva: World Health Organizations, 2010.

GALLASSI, A. D.; ALVARENGA, P. G. D.; ANDRADE, A. G. D.; COUTTOLENC, B. F. The cost of problems caused by alcohol abuse. Rev. Psiquiatr. Clin. (São Paulo), v. 35, p. 6, 2008.

GOTHAM, H.; WHITE, M.; BERGETHON, H.; FEENEY, T.; CHO, D.; KEEHN, B. An implementation story: moving the GAIN from pilot project to statewide use. J. Psychoactive Drugs, v. 40, p. $97-107,2008$.

JOHNSON, J. L. Fundamentals of substance abuse practice. Belmont, CA: Brooks/Cole, 2004.

Recebido para publicação: 02/04/2012

Aceito para publicação: 27/04/2012
LENNOX, R.; DENNIS, M.; SCOTT, C.; FUNK, R. Combining psychometric and biometric measures of substance use. Drug Alcohol Depend., v. 83, p. 95-103, 2006.

PERRY, P.; HEDGES DUROY, T. Adolescent and young adult heroin and non heroin users: a quantitative and qualitative study of experiences in a therapeutic community. J. Psychoactive Drugs, v. 36, p. $75-84,2004$.

RAMCHAND, R.; MORRAL, A.; BECKER, K. Seven-year life outcomes of adolescent offenders in Los Angeles. Am. J. Public Health, v. 99, p. 863-870, 2009.

RILEY, B.; CONRAD, K.; BEZRUCZKO, N.; DENNIS, M. Relative precision, efficiency and construct validity of different starting and stopping rules for a computerized adaptive test: the GAIN substance problem scale. J. App.l Meas., v. 8, p. 48-64, 2007.

SILVEIRA, R. O cuidado de enfermagem e o cateter de Hickman: a busca de evidências. Universidade de São Paulo, Escola de Enfermagem de Ribeirão Preto. 2005

SUBRAMANIAM, G.; STITZER, M.; CLEMMEY, P.; KOLODNER, K.; FISHMAN, M. Baseline depressive symptoms predict poor substance use outcome following adolescent residential treatment. J. Am. Acad. Child Adolesc. Psychiatry, v. 46, p. 10621069, 2007.

TITUS, J.; DENNIS, M.; LENNOX, R.; SCOTT, C. Development and validation of short versions of the internal mental distress and behavior complexity scales in the Global Appraisal of Individual Needs (GAIN). J. Behav. Health Serv. Res., v. 35, p. 195-214, 2008.

WHITE, M. K.; FUNK, R.; WHITE, W.; DENNIS, M. Predicting violent behavior in adolescent cannabis users: The GAIN-CVI. Predicting violent behavior in adolescent cannabis users: The GAIN-CVI. Offender Subst. Abuse Rep., v. 3, p. 67-69, 2003. 